\title{
An Efficient Algorithm for Estimating the Parameters of Multicomponent Cubic Phase Signals
}

\author{
Pengfei Tang, Bin Yuan, Qianqiang Lin, Zengping Chen \\ ATR Key Lab, National University of Defense Technology, Changsha, Hunan Prov., China, 410073 \\ E-mail: tangpengfei@nudt.edu.cn
}

\begin{abstract}
An efficient algorithm is proposed to estimate the parameters of multicomponent cubic phase signals with different amplitudes. This algorithm is based on the product generalized cubic phase function (PGCPF) and the product cubic phase function (PCPF), which are used to compute the cubic phase coefficient and chirp rate of the cubic phase signal, respectively. The parameters of the component with the strongest amplitude are estimated firstly. Then filtering out the strongest component, the parameters of other components in the residual signal are estimated one by one until all of the signal components have been estimated. Computer simulations are carried out to illustrate the effectiveness of the proposed algorithm.
\end{abstract}

Keywords- parameter estimation; cubic phase signal; cubic phase function; generalized cubic phase function

\section{INTRODUCTION}

The polynomial-phase signal (PPS) model is widely used for modelling signals in radar, sonar, biomedicine, communications, etc [1]. One important class of such signal is the cubic phase signal which is frequently encountered in modern radar systems and electronic reconnaissance [2, 3]. This paper focuses on estimating the parameters of multicomponent cubic phase signals.

There are many techniques for estimating the parameters of cubic phase signals. Of these techniques, the maximum likelihood (ML) technique can provide very high estimation accuracy [4]. However, the ML technique requires a threedimensional joint maximization and suffers from heavy computation burden.

Therefore, researchers have advised more computationally efficient alternative approaches based on multilinear functions [5, 6]. The best known multilinear function is the HAF which is designed to estimate the polynomial phase parameters directly [1]. The HAF approach employs iterative differences of the phase to obtain a sinusoid whose frequency is directly proportional to the highest order phase parameter. However, when HAF is applied to multi-component signals, the cross-terms between the components give rise to undesired sinusoids. The product high-order ambiguity function (PHAF) [7], which is the extension of the HAF, has the ability to suppress the noise and spurious harmonics by using high-order multiple transform technique and proper scaling. The disadvantage of the PHAF is the relatively high order of the nonlinearities as the HAF. The higher orders of nonlinearities can produce the higher SNR threshold [8].
The PGCPF is an efficient approach to deal with multicomponent cubic phase signals with the same or similar amplitude [9]. However, when the amplitudes of multiple components are much dissimilar, the weak peaks belonging to the auto-terms may be overshadowed by the peaks caused by undesired cross-terms, and then it will fail to provide reliable estimations.

In this paper, an efficient algorithm is proposed to estimate the parameters of multicomponent cubic phase signals with different amplitudes. The rest of this paper is organised as follows. In Section II, the estimation problem is formulated and the GCPF and PGCPF are briefly reviewed. In Section III, the algorithm that estimates the parameters of multicomponent cubic phase signals is presented. In Section IV, numerical simulations are given to illustrate the presented algorithm. Finally, conclusions are drawn in Section V.

\section{BRIEF REVIEW OF GCPF AND PGCPF}

A multicomponent cubic phase signal embedded in noise is modelled in discrete-time as

$$
\begin{gathered}
x(n)=\sum_{k=1}^{K} A_{k} \exp \left[j\left(a_{k, 0}+a_{k, 1} n+a_{k, 2} n^{2}+a_{k, 3} n^{3}\right)\right]+w(n) \\
-\frac{N-1}{2} \leq n \leq \frac{N-1}{2}
\end{gathered}
$$

where $w(n)$ is the additive complex white Gaussian noise with zero-mean and variance $\sigma^{2}, K$ is the number of signal components, $A_{k}$ is the amplitude of the $k$ th component, $\left\{a_{k, 0}, a_{k, 1}, a_{k, 2}, a_{k, 3}\right\}_{k=1}^{K}$ denotes the phase coefficients to be estimated, and $N$ is an odd integer and the sampling rate is assumed, without loss of generality, to be unity.

GCPF and PGCPF are proposed to estimate the parameters of PPSs with higher order. Due to the lowerorder nonlinearity, they outperform all of the rival algorithms when cubic phase signals are analyzed [9].

\section{A. $G C P F$}

GCPF was originally developed in [9] to estimate the parameters of a PPS. For a cubic phase signal defined as

$$
\begin{aligned}
s(n)= & A \exp \left[j\left(a_{0}+a_{1} n+a_{2} n^{2}+a_{3} n^{3}\right),\right. \\
& -\frac{N-1}{2} \leq n \leq \frac{N-1}{2}
\end{aligned}
$$

The GCPF for $s(n)$ is defined in [9] as follows: 


$$
\begin{aligned}
\operatorname{GCPF}(n, \Omega) & =\sum_{m=0}^{(N-1) / 2} s(n+m) s^{*}(-n+m) s(n-m) \\
& \cdot s^{*}(-n-m) e^{-j 12 n \Omega m^{2}}
\end{aligned}
$$

where $*$ denotes the conjugate.

When the signal in (2) is substituted into (3), the result is

$$
\begin{aligned}
\operatorname{GCPF}(n, \Omega)= & A \exp \left[j\left(4 n a_{1}+4 n^{3} a_{3}\right)\right] \\
& \cdot \sum_{m=0}^{(N-1) / 2} \exp \left[-j 12\left(\Omega-a_{3}\right) n m^{2}\right]
\end{aligned}
$$

It is obvious that in the absence of noise, $\operatorname{GCPF}(n, \Omega)$ yields a peak at $\Omega=a_{3}$. Hence, $a_{3}$ can be estimated as

$$
\hat{a}_{3}=\underset{\Omega}{\arg \max _{\Omega}}|G C P F(n, \Omega)|
$$

After $a_{3}$ has been estimated, the other parameters can be estimated by the dechirping technique [10]

\section{B. $P G C P F$}

The advantage of GCPF is the relatively lower orders nonlinearities involved compared with various rival algorithms, such as the HAF, PHAF, PWVD, HP function [9]. However, the GCPF has fourth-order nonlinearity. It produces cross-terms when multicomponent cubic phase signals are present. The cross-terms in the GCPF have a particular dependence on the time index, while the autoterms are localized along straight lines independent of the time index. Therefore, the cross-terms can be suppressed by the product version of GCPF, which is defined as [9]

$$
\operatorname{PGCPF}(\Omega)=\prod_{l=1}^{L}\left|G C P F\left(n_{l}, \Omega\right)\right|
$$

where $n_{l}$ denotes the $L$ different time positions. For a single component cubic phase signal, the $n$ value which gives rise to the best $a_{3}$ estimation is $n=(N-1) / 4$. For multicomponent cubic phase signals, in order to attenuate the cross-terms and enhance the auto-terms, $n_{l}$ should be around $(N-1) / 4$ and sufficiently spaced.

\section{C. limitations of $P G C P F$}

When dealing with multicomponent cubic phase signals using PGCPF, it is generally assumed that all the components have the same or similar amplitude [9], which may not be true in practice. If the amplitudes of multiple components are much different, PGCPF will fail to provide reliable estimations. This is because the weak peaks belonging to the auto-terms may be overshadowed by the peaks caused by undesired cross-terms.

Two examples will be given to illustrate the shortcomings of PGCPF. In these examples, we assume that $N=513$ samples of data are available. The signals conform to the model as shown in (1). Specifically, the SNR is defined as the first component's SNR, which is $A_{1}^{2} / \sigma^{2}$. In these examples, SNR is set to be $0 \mathrm{~dB}$.

Fig. 1 shows the amplitude of GCPF for the sum of two cubic phase signals with the same amplitude. The first component has parameters $a_{1,1}=\pi / 6, a_{1,2}=1 \times 10^{-3}$, $a_{1,3}=3 \times 10^{-6}, A_{1}=1$, while the second component has parameters $a_{2,1}=\pi / 4, a_{2,2}=3 \times 10^{-3}, a_{2,3}=5 \times 10^{-6}$, $A_{2}=1$. For simplicity, the phase coefficients $a_{1,0}$ and $a_{2,0}$ are assumed to be zero. From Fig. 1, it is shown that the auto-terms are submerged in the cross-terms and they can not be detected correctly.

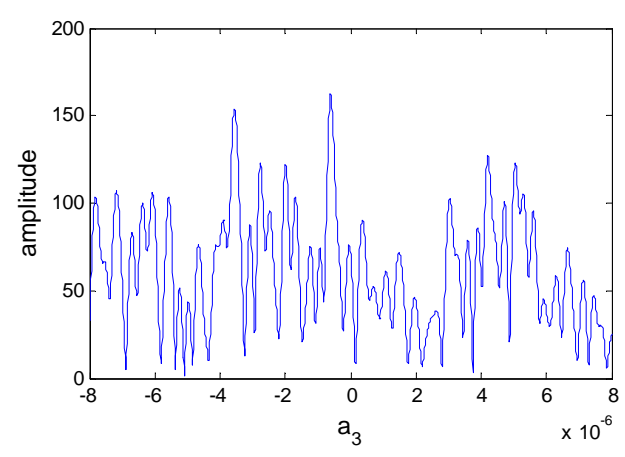

Figure 1. GCPF for components with the same amplitude

Fig. 2 shows the amplitude of PGCPF with the time positions given by: [ $n_{1}=108, n_{2}=118, n_{3}=128$, $\left.n_{4}=138, n_{5}=148\right]$. It is obvious that the cross-terms are attenuated and auto-terms are amplified greatly. The cubic phase coefficients $a_{1,3}$ and $a_{2,3}$ corresponding to the two signals can be easily obtained from Fig. 2 .

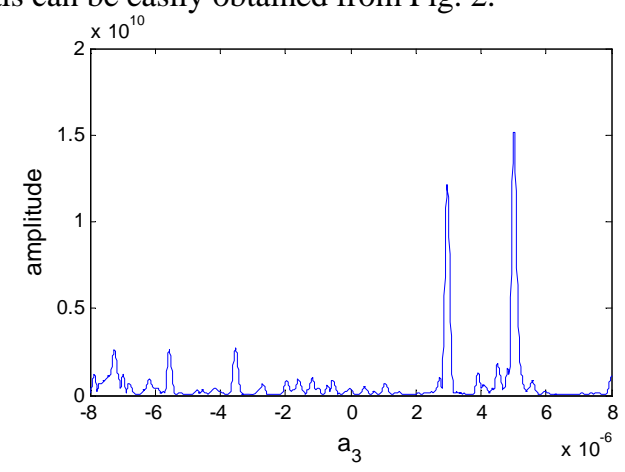

Figure 2. PGCPF for components with the same amplitude

When the amplitudes of two components are different: $A_{1}=0.4$ and $A_{2}=1$, using the same time positions as in Fig. 2, the amplitude of PGCPF is shown in Fig. 3. We can clearly see only one peak corresponding to the component with the stronger amplitude. This is because the difference in the amplitudes is amplified in the PGCPF. 


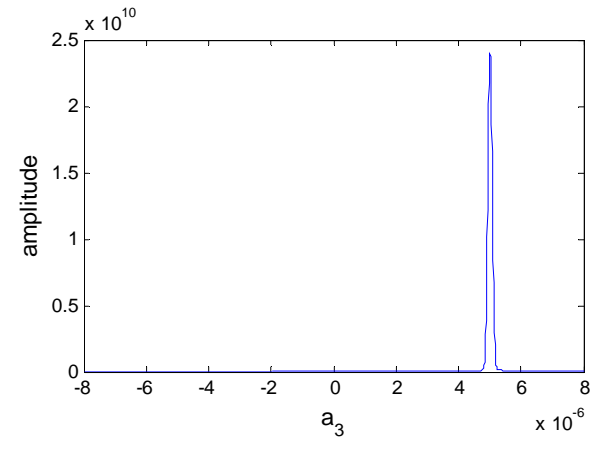

Figure 3. PGCPF for components with different amplitudes

\section{ALGORITHM FOR PROCESSING MULTICOMPONENT CUBIC PHASE SIGNALS}

Even though PGCPF has the advantage to suppress noise and cross-terms, it can only reveal the component with higher amplitude. The estimation algorithm for multicomponent cubic phase signals with different amplitudes is presented as follows.

Step 1) Initialize by setting $k=1$ and $x_{k}(n)=x(n)$, for $-\frac{N-1}{2} \leq n \leq \frac{N-1}{2}$.

Step 2) Estimate the parameters of the $k$ th component, which has the highest amplitude.

a) Let $x_{k}^{(3)}(n)=x_{k}(n)$, for $-\frac{N-1}{2} \leq n \leq \frac{N-1}{2}$.

b) Choose a set of integer $n_{l}$ and compute $\operatorname{PGCPF}(\Omega)$. Then estimate $a_{k, 3}$ by

$$
\hat{a}_{k, 3}=\arg \max _{\Omega}(P G C P F(\Omega))
$$

c) Dechirp the original signal by $\hat{a}_{k, 3} n^{3}$, and then estimate $\hat{a}_{k, 2}$ using PCPF [11]:

$$
\begin{aligned}
x_{k}^{(2)}(n) & =x_{k}^{(3)}(n) \exp \left(-j \hat{a}_{k, 3} n^{3}\right), \\
\hat{a}_{k, 2} & =\arg \max (\operatorname{PCPF}(\Omega))
\end{aligned}
$$

where $\operatorname{PCPF}(\Omega)=\prod_{q=1}^{Q}\left|\sum_{m=0}^{(N-1) / 2} x_{k}^{2}\left(n_{q}+m\right) x_{k}^{2}\left(n_{q}-m\right) e^{-j \Omega m^{2}}\right|$. In order to suppress the cross-terms and reduce the computational cost, $n_{q}$ should be around $(N-1) / 2$ and sufficiently spaced.

d) Estimate $\hat{a}_{k, 1}$ by dechirping the original signal by $\hat{a}_{k, 2} n^{2}+\hat{a}_{k, 3} n^{3}$ and then using the Fourier transform:

$\hat{a}_{k, 1}=\arg \max _{\Omega}\left(\sum_{n=-(N-1) / 2}^{(N-1) / 2} x_{k}^{(3)}(n) \exp \left[-j\left(\hat{a}_{k, 2} n^{2}+\hat{a}_{k, 3} n^{3}+j \Omega n\right)\right]\right)$

e) Estimate $\hat{a}_{k, 0}$ and $A_{k}$ by evaluating

$$
x_{k}^{(0)}(n)=x_{k}^{(3)}(n) \exp \left[-j\left(\hat{a}_{k, 1} n+\hat{a}_{k, 2} n^{2}+\hat{a}_{k, 3} n^{3}\right)\right]
$$

$$
\begin{aligned}
& \hat{a}_{k, 0}=\text { angle }\left\{\sum_{n=-(N-1) / 2}^{(N-1) / 2} x_{k}^{(0)}(n)\right\} \\
& A_{k}=\frac{1}{N}\left|\sum_{n=-(N-1) / 2}^{(N-1) / 2} x_{k}^{(0)}(n)\right|
\end{aligned}
$$

Step 3) Filtering out the $k$ th signal component

a)

$$
\begin{gathered}
\bar{x}_{k}^{(0)}(n)=x_{k}^{(0)}(n)-\frac{1}{N} \sum_{n_{1}=-(N-1) / 2}^{(N-1) / 2} x_{k}^{(0)}\left(n_{1}\right) \\
\text { for }-\frac{N-1}{2} \leq n \leq \frac{N-1}{2}
\end{gathered}
$$

b)

$$
x_{k+1}(n)=\bar{x}_{k}^{(0)}(n) \exp \left[j\left(\hat{a}_{k, 1} n+\hat{a}_{k, 2} n^{2}+\hat{a}_{k, 3} n^{3}\right)\right]
$$

Step 4) Substitute $k=k+1$, and go back to Step 2 until all of the signal components have been estimated or the residual energy of the signal is less than a threshold $\varepsilon E_{x}$, where $\varepsilon$ is an arbitrarily small fraction, and $E_{x}$ is the energy of the original signal.

At the end of Step 2, the parameters of the $k$ th component have been estimated. Its residual content will be highly localized around the $0 \mathrm{~Hz}$ spectral region [12, 13]. It is able to efficiently remove this component via Step 3 [14]. In Step 3, the low frequency content is filtered out from the dechirped signal, and then the outcome is modulated by $\exp \left[j\left(\hat{a}_{k, 1} n+\hat{a}_{k, 2} n^{2}+\hat{a}_{k, 3} n^{3}\right)\right]$.

\section{NumERICAL SimUlations}

In this section, numerical simulations are provided to illustrate the performance of the proposed approach.

The signal to be analyzed is the same as in Fig. 3. The amplitude of PGCPF of original signal $x(n)$ is shown in Fig. 4(a) where a clear peak can be seen. Then the cubic phase parameter $a_{2,3}$ corresponding to the stronger component is estimated. $x_{1}(n)$ is obtained by dechirping the original signal by $\hat{a}_{2,3} n^{3}$. The amplitude of PCPF of $x_{1}(n)$ is shown in Fig. 4(b), where the time positions are given by: [ $n_{1}=236, n_{2}=256, n_{3}=276$ ]. Then the quadratic phase parameter $a_{2,2}$ is estimated. Dechirp $x_{1}(n)$ by $\hat{a}_{2,2} n^{2}$, and then $x_{2}(n)$ is obtained. The amplitude of FFT for $x_{2}(n)$ is shown in Fig. 4(c). In order to obtain accurate peak location, the FFT operation is implemented by padding zeros to length 2048. From Fig. 4(c), the initial frequency $a_{2,1}$ can be estimated. After the parameters of the first component are estimated, we remove it from the original signal. Next, we compute PGCPF, PCPF and FFT of the residual signal and then the parameters of the weaker component can be estimated.

Table 1 shows the estimation bias and the mean squared error (MSE) of the phase parameter estimations obtained from 200 independent Monte-Carlo simulations. The MSE is compared with the CRLB, which is given in [15]. 
Table 1. The estimation bias, MSE and CRLB

\begin{tabular}{|lccc|}
\hline & $a_{2,3}$ & $a_{2,2}$ & $a_{2,1}$ \\
\hline True & $5 \times 10^{-6}$ & $3 \times 10^{-3}$ & $\pi / 4$ \\
estimation bias & $4.9900 \times 10^{-6}$ & $3.0050 \times 10^{-3}$ & 0.7854 \\
MSE (dB) & -157.60 & -100.24 & -50.26 \\
& $a_{1,3}$ & $a_{1,2}$ & $a_{1,1}$ \\
True & $3 \times 10^{-6}$ & $1 \times 10^{-3}$ & $\pi / 6$ \\
estimation bias & $2.9800 \times 10^{-6}$ & $1.0150 \times 10^{-3}$ & 0.5216 \\
MSE (dB) & -153.98 & -96.48 & -53.79 \\
& & & \\
CRLB (dB) & -158.25 & -115.96 & -65.56 \\
\hline
\end{tabular}

It is shown that the signal component with stronger amplitude has higher estimation accuracy than the other component. This is because the removing operation causes error propagation in the recursive procedure.
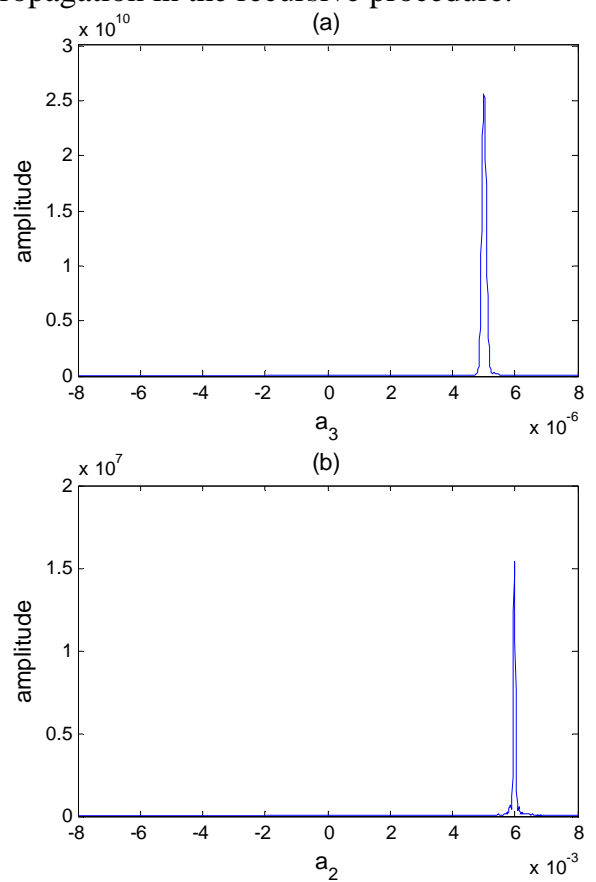

(c)

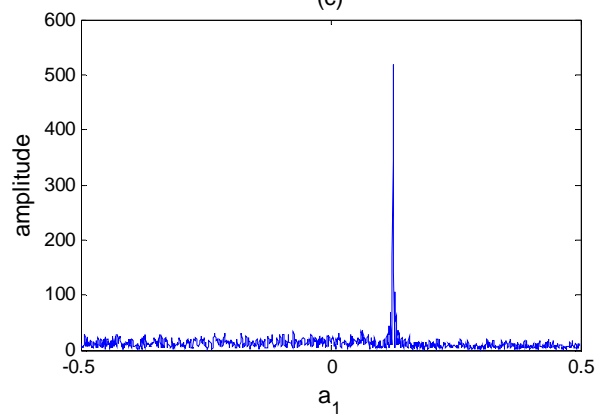

Figure 4. The amplitudes of transformations. (a) PGCPF of the original signal, (b) PCPF of the original signal, (c) FFT of the original signal

\section{CONCLUSION}

An efficient algorithm for estimating the parameters of multicomponent cubic phase signals with different amplitudes has been proposed. It starts by estimating the parameters of the signal component with the strongest amplitude. Then removing the signal component whose parameters have been estimated, it proceeds to estimate the next signal component, and so on, until all of the signal components have been estimated. In additional, it has been shown that the PGCPF and PCPF used in the algorithm could suppress the noise and cross-terms significantly. Numerical simulations validate the performance of the proposed algorithm.

\section{REFERENCES}

[1] S. Peleg, and B. Porat. "Estimation and classification of polynomialphase signals”, IEEE Transactions on Information Theory, volume 37, pp. 422-430, 1991.

[2] Yong Wang and Yicheng Jiang. "Inverse synthetic aperture radar imaging of maneuvering target based on the product generalized cubic phase function", IEEE Geoscience and remote sensing Letters, volume 8, pp. 958-962, 2011

[3] P. O'Shea. "A fast algorithm for estimating the parameters of a quadratic FM signal”, IEEE Trans. Signal Process., volume AES-22, pp. 708-715, 1986.

[4] T. Abotzoglou. "Fast maximum likelihood joint estimation of frequency and frequency rate”, IEEE Trans. Aerosp. Electron. Syst., volume 52, pp. 385-393, 1986.

[5] B. Boashash, P. O'Shea. "Polynomial Wigner-Ville distributions and their relationship to time-varying higher order spectra”, IEEE Trans. Signal Process., volume 42, pp. 216-220, 1994.

[6] P.Wang, I. Djurovic, and J. Yang. "Generalized high-order phase function for parameter estimation of polynomial phase signal”, IEEE Trans. Signal Process., volume 54, pp. 3023-3028, 2008.

[7] S. Barbarossa, A. Scaglione, and G. Giannakis. "Product high-order ambiguity function for multicomponent polynomial phase signal modelling”, IEEE Trans. Signal Process., volume 46, pp. 691-708, 1998.

[8] Muhammad Z. Ikram, G. Tong Zhou. "Estimation of multicomponent polynomial phase signals of mixed orders”, Signal Processing, volume 81, pp. 2293-2308, 2001.

[9] P. O'Shea and R. A. Wiltshire. "A new class of multilinear functions for polynomial phase signal analysis”, IEEE Trans. Signal Process., volume 57, pp. 2096-2109, 2009.

[10] S. Peleg and B. Friedlander. "The discrete polynomial phase transform”, IEEE Trans. Signal Process., volume 43, pp. 1901-1914, 1995.

[11] Pu Wang, Jianyu Yang. "Multicomponent chirp signals analysis using product cubic phase function”, Digital Signal Processing, volume 16, pp. 654-666, 2006.

[12] P. O'Shea. "On refining polynomial phase signal parameter estimates”, IEEE Transactions on Aerospace and Electronic Systems, volume 46, pp. 978-987, 2010.

[13] S. Peleg, B. Friedlander. "Multicomponent signal analysis using the polynomial-phase transform”, IEEE Transactions on Aerospace and Electronic Systems, volume 32, pp. 378-387, 1996.

[14] M. Faquharson, P. O'Shea. "Extending the performance of the cubic phase function”, IEEE Trans. Signal Process., volume 55, pp. 47674774, 2007.

[15] S. Peleg, B. Porat. "The Cramer-Rao lower bound for signals with constant amplitude and polynomial phase”, IEEE Trans. Signal Process., volume 39, pp. 749-752, 1991. 